\title{
Endoscopic-Assisted Microsurgical Resection of Right Recurrent Meckel's Cave Meningioma Extended to Cavernous Sinus
}

\author{
Arianna Fava ${ }^{1}$ Paolo di Russo ${ }^{1}$ Lorenzo Giammattei ${ }^{2}$ Sébastien Froelich ${ }^{2}$ \\ ${ }^{1}$ Department of Neurosurgery, Lariboisiere Hospital, Paris, France \\ ${ }^{2}$ Department of Neurosurgery, Lariboisiere Hospital, University of \\ Address for correspondence Arianna Fava, MD, Department of \\ Paris Diderot, Paris, France \\ Neurosurgery, Lariboisiere Hospital, Paris 75010, France \\ (e-mail: arianna.f.90@gmail.com).
}

J Neurol Surg B Skull Base 2022;83(suppl S3):e632-e634.

\begin{abstract}
Keywords

- cavernous sinus

- meningioma

- endoscopic assisted

- pterygopalatine fossa

- infratemporal fossa
\end{abstract}

Objective This study was aimed to present the complete removal of a large recurrent Meckel's cave meningioma.

Design This study is a case report.

Setting The study was conducted at Department of Neurosurgery and Skull Base Laboratory at Lariboisiére Hospital, Paris.

Participant A 53-year-old male was presented with a severe V1, V2, and V3 hypoesthesia and pain. He was operated 7 years ago for a right Meckel's cave meningioma with postoperative $\mathrm{V} 1-\mathrm{V} 2$ hypoesthesia. Magnetic resonance imaging (MRI) showed a large tumor recurrence extending into the cavernous sinus (CS), posterior fossa (PF), sphenoid sinus (SS), pterygopalatine (PPF), and infratemporal fossa (ITF; - Fig. 1).

Main Outcome Measures Radiological results and postoperative course were assessed for this study.

Results The previous right frontotemporal approach was used. The lateral wall of the orbit, the middle fossa floor and the anterior temporal base were drilled to expose the

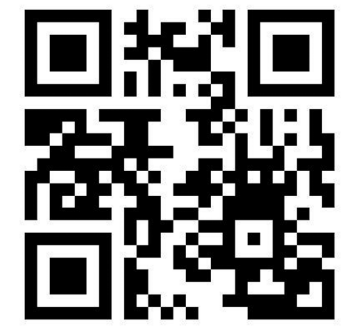

received

April 2, 2020

accepted

December 1, 2020

published online

May 11, 2021 www.thieme.com/skullbasevideos

www.thieme.com/jnlsbvideos

(c) 2021. The Author(s).

This is an open access article published by Thieme under the terms of the Creative Commons Attribution-NonDerivative-NonCommercial-License, permitting copying and reproduction so long as the original work is given appropriate credit. Contents may not be used for commercial purposes, or adapted, remixed, transformed or built upon. (https://creativecommons.org/ licenses/by-nc-nd/4.0/)

Georg Thieme Verlag KG, Rüdigerstraße 14, 70469 Stuttgart, Germany 
orbit, PPF, and ITF. Foramen ovale (FO), foramen rotondum (FR), and superior orbital fissure (SOF) were opened. The meningoorbital band was cut and the lateral wall of CS was elevated (-Fig. 2). The inferior orbital fissure was opened and tumor removed into the ITF, PPF, and orbit. After entering Meckel's cave from above, tumor was removed from PF. After microsurgical tumor removal, a 45-degree endoscope was used to remove tumor remnant and mucosa into SS. A watertight dural closure with pericranium was performed, reinforced with autologous fat and fibrin glue. Postoperative MRI showed complete tumor resection (-Fig. 1). The patient experienced a right-side keratitis that resolved within 10 days and a V3 hypoesthesia that improved at 2 months. Conclusion This surgical case shows how the anatomical knowledge is mandatory in skull base surgery and how the integration of microsurgical and endoscopic-assisted techniques allows to obtain optimal results.

The link to the video can be found at: https://youtu.be/qxt_389AdWU.

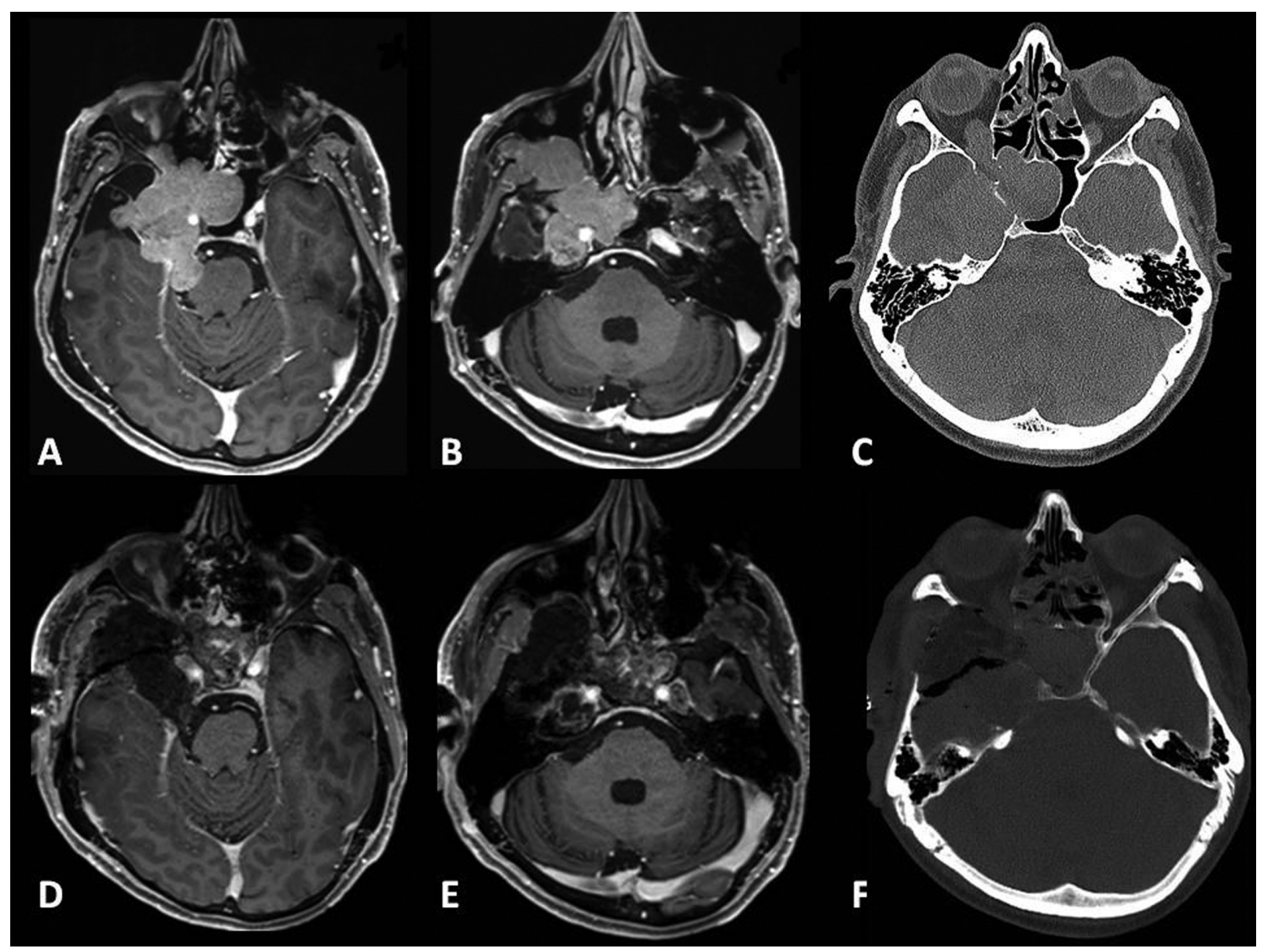

Fig. 1 Pre- and postoperative images. (A) Preoperative axial T1-weighted gadolinium-enhanced magnetic resonance imaging (MRI) showing the meningioma extending into the posterior fossa (PF), cavernous sinus (CS), orbit and sphenoid sinus (SS). (B) Tumor extension to middle fossa, pterygopalatine (PPF) and infratemporal fossa (ITF). (C) Preoperative axial computed tomography (CT) scan showing the greater sphenoid wing erosion and the involvement of the SS. (D, E) Postoperative axial T1-weighted gadolinium enhanced MRI showing complete tumor resection. (F) Postoperative axial CT scan showing the fat graft used for closure. 

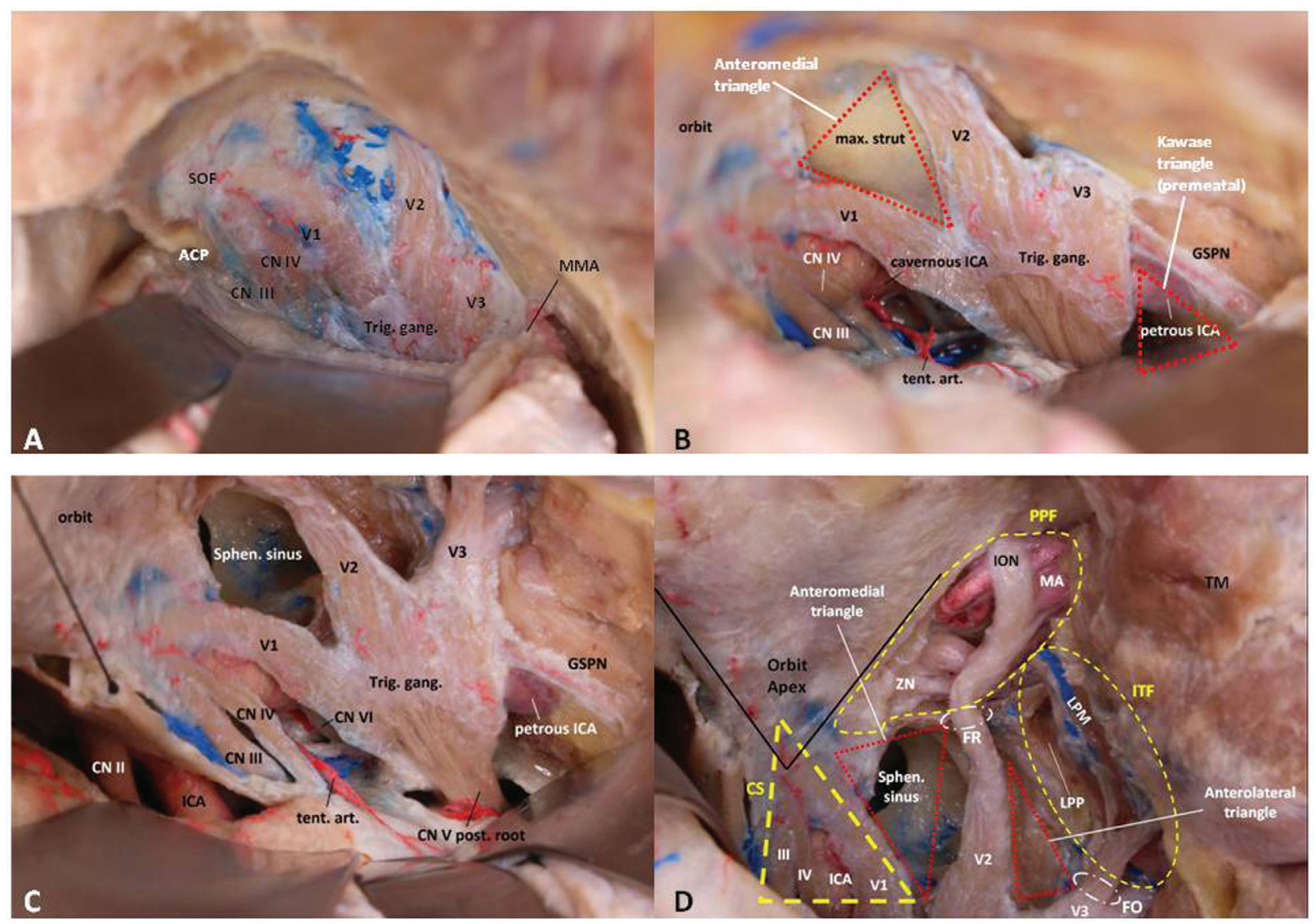

Fig. 2 Step-by-step cadaveric dissection of the cavernous sinus and middle fossa exposure. (A) Peeling of the lateral wall of the cavernous sinus showing cranial nerve (CN) III, IV, and V1 entering into the superior orbital fissure (SOF) and V2, V3. The middle meningeal artery (MMA), posterior to V3, exits from foramen spinosum. (B) Exposure of the anteromedial triangle between V1 and V2 and petrous apex drilling exposing the petrous internal carotid artery (ICA). The superior wall of Meckel's cave is removed. (C) Sphenoid sinus (SS/Sphen. sinus) exposure after drilling the anteromedial triangle (V1-V2). (D) Final exposure after drilling the anterolateral triangle (V2-V3) and opening foramen rotondum (FR), foramen ovale (FO). The pterygopalatine fossa (PPF) and the full course of the maxillary nerve are exposed until it reaches the infraorbital groove. ACP, anterior clinoid process; GSPN, greater superficial petrosal nerve; ICA, internal carotid artery; ION, infraorbital nerve; ITF, infratemporal fossa; LPM, lateral pterygoid muscle; LPP, lateral pterygoid plate; MA, maxillary artery; max. strut, maxillary strut; post. root, posterior root; TM, temporal muscle; tent. art., tentorial artery (Bernasconi-Cassinari); Trig. gang., trigeminal ganglion; ZN, zygomatic nerve.

\section{Conflict of Interest}

None declared. 